\title{
The shift of macrophages toward M1 phenotype promotes aortic valvular calcification
}

\author{
Geng Li, MD, PhD, ${ }^{\mathrm{a}}$ Weihua Qiao, MD, PhD, ${ }^{\mathrm{a}}$ Wenjing Zhang, MD, ${ }^{\mathrm{b}}$ Fei Li, MD, ${ }^{\mathrm{a}}$ Jiawei Shi, MD, PhD, ${ }^{\mathrm{a}}$ \\ and Nianguo Dong, $\mathrm{MD}, \mathrm{PhD}^{\mathrm{a}}$
}

\begin{abstract}
Objective: The purpose of the present study was to comprehensively compare the phenotype profile of infiltrated macrophages in human noncalcified and calcific aortic valves, and to determine whether the shift of macrophage polarization modulates valvular calcification in vitro.
\end{abstract}

Methods: Cell surface markers of macrophages and inflammatory cytokines expression in 90 cases of human noncalcified and calcific aortic valve leaflets were analyzed. The normal aortic valve interstitial cells were isolated and cultured in vitro. After incubation with nonconditioned medium and conditioned medium from unstimulated or lipopolysaccharide-stimulated U937 monocytes, valve interstitial cells were evaluated by osteogenic differentiation markers.

Results: Infiltration of macrophages was enhanced in the calcific aortic valves, and M1 phenotype was the predominant macrophage subsets. In addition, both proinflammatory and anti-inflammatory cytokines were significantly upregulated in the calcific aortic valves. Furthermore, lipopolysaccharide-stimulated monocytes presented with increased expression of inducible nitric oxide synthase and high proportional CD11c-positive (M1) macrophages. Conditioned medium from unstimulated monocytes promoted the osteogenic differentiation of valve interstitial cells in vitro, as evidenced by increased markers such as bone morphogenetic protein 2, osteopontin, and alkaline phosphatase. Conditioned medium from M1 macrophages further enhanced valve interstitial cells calcification. Enzyme-linked immunosorbent assay showed that M1 phenotype macrophages secreted tumor necrosis factors $\alpha$ and interleukin 6 , and neutralizing antibodies to these 2 proinflammatory cytokines attenuated induction of osteogenic differentiation and calcification by the conditioned media.

Conclusions: Both total numbers and polarization of macrophage influence the process of calcification in human aortic valve. The shift toward M1 phenotype might promote valve interstitial cell calcification. (J Thorac Cardiovasc Surg 2017;153:1318-27)

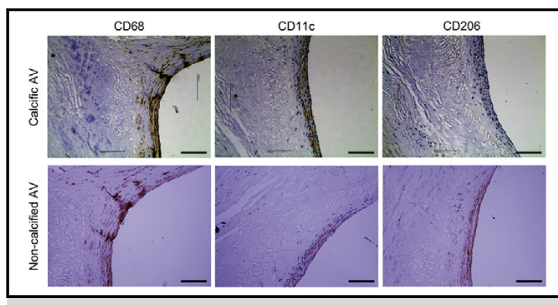

Macrophages infiltration in calcific and noncalcified aortic valves.

\section{Central Message}

Macrophage infiltration and polarization influence the process of calcification in human aortic valves.

\section{Perspective}

Macrophages are one of the most important cells in valvular calcification. The shift of macrophage toward M1 phenotype promotes this pathophysiologic process. Targeting macrophage polarization and altering their phenotype to adapt to the microenvironment might be promising for therapy of CAVD.

See Editorial Commentary page 1328.
Calcific aortic valve disease (CAVD) is the third leading cause of cardiovascular diseases, resulting in more than 23,000 deaths annually in the United Sates. ${ }^{1}$ With the rapid

From the Departments of ${ }^{\mathrm{a}}$ Cardiovascular Surgery and ${ }^{\mathrm{b}}$ Ultrasound, Union Hospital, Tongji Medical College, Huazhong University of Science and Technology, Wuhan, People's Republic of China.

This work was supported by the National Natural Science Foundation of China (81270297 and 81300175).

The protocol of this study was approved by the Ethical Committee of Tongji Medical College, Huazhong University of Science and Technology (Date: March 5, 2012; No: S194) and conducted in accordance with the ethical standards laid down in the Declaration of Helsinki.

G.L. and W.Q. are co-first authors who contributed equally to this work.

Received for publication April 13, 2016; revisions received Dec 31, 2016; accepted for publication Jan 25, 2017; available ahead of print March 7, 2017. aging of society, the incidence of CAVD is increasing in China. ${ }^{2}$ Valvular calcification is a major characteristic pathologic feature of CAVD. ${ }^{3,4}$ Inflammation seems to play an 

Abbreviations and Acronyms
ALP = alkaline phosphatase
$\mathrm{BMP}=$ bone morphogenetic protein
CAVD $=$ calcific aortic valve disease
IL = interleukin
LPS = lipopolysaccharide
OPN = osteopontin
PBS = phosphate-buffered saline
$\mathrm{PCR}=$ polymerase chain reaction
RPMI $=$ Roswell Park Memorial Institute
$\mathrm{TGF}=$ transforming growth factor
$\mathrm{TNF}=$ tumor necrosis factor
VIC $=$ valvular interstitial cell

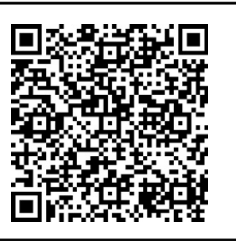

essential role in the development of aortic valve leaflet calcification according to pathologic examinations..$^{5-7}$ Studies have indicated that CAVD is an inflammatory process involving infiltration of a number of cell types encompassing lymphocytes, plasma cells, mast cells, histiocytes, mast cells, and macrophages. ${ }^{6,7}$

Innate immune cells (eg, monocytes and macrophages) have not been well studied in CAVD. Abundant infiltration of macrophages has been observed in explanted human valves associated with extensively tissue remodeling and neovascularization. ${ }^{8,9}$ Macrophages have at least 2 distinct states of polarized activation: the classically activated (M1) phenotype (related to proinflammation) and the alternatively activated (M2) phenotype (related to pro-healing). ${ }^{10}$ It is reported that M1 and M2 could exist in the central places of atherosclerotic and calcific lesions, and convert to each other in response to microenvironmental changes. ${ }^{11,12}$ The ratio of M1/M2 subsets was more related to atherosclerotic plaque stability and progression rather than the total number of macrophages. ${ }^{13,14}$ In addition, CAVD has similar pathologic processes and clinical risk factors to atherosclerosis. ${ }^{15}$ Therefore, it was assumed that CAVD might be evoked not only by a sustained proinflammatory response but also by the imbalance of M1 and M2 macrophages polarization.

To date, however, the possible role of varied phenotypes of macrophages in CAVD is poorly understood. Because valvular interstitial cells (VICs) are the main cell population in heart valve leaflets, it has been considered that there are cellular interactions between VICs and macrophages, and macrophage polarization affects proinflammatory cytokine release on VIC activation and osteogenic differentiation. In the present study, we hypothesized that macrophages infiltration and polarization regulate VIC calcification. We analyzed cell surface markers of macrophages and cytokine expression in noncalcified or calcific aortic valve leaflet obtained by heart transplant programs and cardiovascular surgery. Furthermore, normal primary VICs isolated from noncalcified aortic valve tissue were cultured in vitro and incubated with the conditioned medium from unstimulated or lipopolysaccharide (LPS)-stimulated U937 monocytic cells to elucidate functions of M1 macrophage phenotype on VICs. This study may give us more information about the macrophage subsets and their functions in CAVD.

\section{MATERIALS AND METHODS}

\section{Patient Selection and Specimen Acquisition}

The protocol of this study was approved by the Ethical Committee of Tongji Medical College affiliated to Huazhong University of Science and Technology, P. R. China, and conducted in accordance with the ethical standards stated in the Declaration of Helsinki. Informed consent was obtained from all patients. Human aortic valves were obtained from 90 patients at the time of aortic valve replacement or heart transplantation. On the basis of the gross appearance and echocardiography, the levels of calcification of the aortic valve were classified as follows: grade 0 , no calcification; grade 1 , sclerosis or mild calcification; grade 2, moderate calcification; and grade 3 , severe calcification (Table 1). Calcific aortic valves (calcification grade 1 or 2) were obtained from 60 patients with CAVD during aortic valve replacement. Control noncalcified aortic valves (calcification grade 0) were collected from the explanted hearts of 30 patients without CAVD undergoing heart transplantation. Valves with calcification grade 3 , which were distorted by multiple nodular deposits and loss of cells, were excluded. Exclusion criteria also included rheumatic disease, infective endocarditis, bicuspid aortic valves, and valves with moderate to severe aortic valve regurgitation. All patients underwent a comprehensive examination by an experienced echocardiographer.

\section{Immunohistochemical Staining}

Each aortic valve excised at the time of surgery was placed in a container filled with cold phosphate-buffered saline (PBS). One cusp was immediately frozen in liquid nitrogen. One cusp was fixed in $4 \%$ paraformaldehyde, dehydrated, and subsequently embedded in paraffin Immunohistochemical staining was performed as previously described. ${ }^{16}$ After antigen retrieval, deparaffinized sections of each valve were incubated overnight with mouse monoclonal antihuman CD68 (1:100, Abcam, Cambridge, UK), mouse monoclonal anti-human CD11c (1:100, Abcam), and mouse monoclonal anti-human CD206 (1:200, Abcam) followed by application of a secondary antibody (biotinylated anti-mouse immunoglobulin-G). The numbers of immunopositive cells were counted in 6 matched microscope fields at $400 \times$ magnifications.

\section{Quantitative Real-Time Polymerase Chain Reaction}

The RNA was isolated and reversely transcribed to cDNA as previously described. ${ }^{16}$ Primers were purchased from GenScript Co, Ltd (Nanjing, China). Sequences for all quantitative PCR primers are shown in Table E1. Quantitative real-time PCR reaction was performed on the ABI 7900HT Sequence Detection System (Applied Biosystems, Carlsbad, Calif). Relative gene expression was normalized to $\beta$-actin level. The delta/delta $\mathrm{Ct}$ calculation method was used for analyzing the data. 
TABLE 1. Taxonomy of valvular calcification grade

\begin{tabular}{llll}
\hline \multicolumn{1}{c}{ Grade } & \multicolumn{1}{c}{ Gross appearance } & Echocardiographic imaging \\
\hline 0 & No calcification & Normal morphology, translucent or opalescent, $<1 \mathrm{~mm}$ thick, pliable & No calcific nodule \\
1 & Sclerosis/mild & Irregular, nonuniform thickening of portions of the aortic valve, fine & Focal areas of leaflet thickening, small isolated \\
& calcification & stippled calcific nodules, stiffening & Multiple larger calcific nodules (diameter 2-7 mm) \\
2 & Moderate calcification & Larger complex plates and nodules & Extensive thickening, calcification of all cusps, \\
3 & Severe calcification & Extensive thickening and bulky calcific nodules effacing leaflet & bulky calcific nodules (diameter $>7 \mathrm{~mm}$ ) \\
& & architecture, ulceration of calcific nodules & \\
\hline
\end{tabular}

\section{Valvular Interstitial Cell Isolation and U937 Monocyte Cell Culture}

Human VICs were isolated from noncalcified aortic valves obtained from patients undergoing heart transplantation using collagenase digestion as previously described. ${ }^{16}$ Interstitial cells were cultured in Dulbecco's modified Eagle medium containing penicillin G, streptomycin, and $10 \%$ fetal bovine serum at $37^{\circ} \mathrm{C}$ and $5 \% \mathrm{CO}_{2}$. The cells from passages 3 to 5 were used for the experiments. U937 monocytic cells were purchased from the cell center of Wuhan University and cultured in Roswell Park Memorial Institute (RPMI) 1640 medium containing 10\% fetal bovine serum, penicillin, and streptomycin. For differentiation to the adherent macrophages, cells were diluted to a concentration of $5.0 \times 10^{5} / \mathrm{mL}$ and treated by phorbol-12-myristate-13-acetate at a concentration of $100 \mathrm{ng} / \mathrm{mL}$ for 24 hours. The medium was replaced with fresh RPMI 1640 before the cells were treated with medium alone or medium containing $100 \mathrm{ng} / \mathrm{mL}, 200 \mathrm{ng} /$ $\mathrm{mL}$, or $500 \mathrm{ng} / \mathrm{mL}$ of LPS for 12 hours.

\section{Conditioned Medium Harvest and Valvular Interstitial Cell Calcification Model}

After treatment with LPS, U937 cells were washed thoroughly and then placed in fresh RPMI 1640 media. After 48 hours of incubation, U937 cells were collected for RNA and protein extraction, and the supernatant was collected and used as conditioned medium. To ensure that the effect of conditioned media on VICs was not due to the direct effect of LPS carried over from the treated cells, the conditioned medium was incubated with polymyxin $\mathrm{B}$ for 1 hour at $37^{\circ} \mathrm{C}$ to neutralize LPS before application on VICs co-culture. ${ }^{17}$

To provoke calcification, VICs were treated as previously described. ${ }^{18}$ Briefly, VICs at a concentration of $5.0 \times 10^{5}$ cells $/ \mathrm{mL}$ were incubated with the procalcifying medium containing Dulbecco's modified Eagle medium, $5 \%$ fetal bovine serum, $50 \mu \mathrm{g} / \mathrm{mL}$ ascorbic acid, $100 \mathrm{nmol} / \mathrm{L}$ dexamethasone, and $10 \mathrm{mmol} / \mathrm{L} \beta$-glycerophosphoric acid. To investigate the effect of macrophage on the osteogenic differentiation of human VICs, $1 \mathrm{~mL}$ nonconditioned (fresh) medium or conditioned medium from unstimulated or LPS-stimulated macrophages as described earlier was added to the VICs procalcifying medium. Neutralizing antibodies to tumor necrosis factor (TNF)- $\alpha$ and interleukin (IL)-6 were obtained from eBioscience (San Diego, Calif). After 7 days of incubation, the cultured cells were collected for RNA and protein extraction.

\section{Flow Cytometry}

Phorbol-12-myristate-13-acetate-maturated, untreated, or LPS-treated cells were collected and washed with PBS. Cells were resuspended into $5.0 \times 10^{5}$ cells/tube and washed twice with PBS containing $1 \%$ bovine serum albumin. Then, cells were incubated with $3 \mu \mathrm{L}$ of fluorescein isothiocyanate-conjugated monoclonal antibody against $\mathrm{CD} 11 \mathrm{c}$ (eBioscience) for 30 minutes at $37^{\circ} \mathrm{C}$ in the dark. The samples were analyzed by a flow cytometer (BD Immunocytometry Systems, San Jose, Calif).

\section{Enzyme-Linked Immunosorbent Assay}

Cell culture medium was collected after treatment with LPS or control medium. TNF- $\alpha$ and IL- 6 in the conditioned media were measured by enzyme-linked immunosorbent assay according to the manufacturer's instructions (Wuhan Boster Biological Technology Ltd, Wuhan, China).

\section{Western Blotting}

Protein expression was demonstrated by Western blotting using specific antibodies against inducible nitric oxide synthase (1:1000, Abcam), bone morphogenetic protein (BMP)2 (1:1000, Abcam), osteopontin (OPN) (1:1000, Abcam), alkaline phosphatase (ALP) (1:1000, Abcam), and glyceraldehyde 3-phosphate dehydrogenase following standard protocols as previously described. ${ }^{19}$

\section{Alizarin Red Staining}

Alizarin red staining for calcium deposits was performed as described previously. ${ }^{16}$ Briefly, VICs were washed twice with PBS and fixed for 15 minutes in $4 \%$ paraformaldehyde. After incubation with $0.2 \%$ alizarin red solution for 30 minutes, excess dye was removed by washing with distilled water.

\section{Statistical Analysis}

Continuous data were expressed as mean \pm standard deviation. The differences between 2 groups were assessed by the Student $t$ test, and multiple comparisons between more than 2 groups were conducted by 1-way analysis of variance. Categoric data were expressed as a percentage and compared with the chi-square test. Statistical analysis was carried out using GraphPad Prism 5 software (GraphPad Software, La Jolla, Calif).

\section{RESULTS}

\section{Patient Demographics}

The demographic data of patients are summarized in Table E2. Significant differences between patients with and without CAVD were seen for age, body mass index, hypertension, coronary artery disease, and statin use. The groups did not differ with regard to sex, serum lipid profile, and serum creatinine concentration. As for medication, statin treatment in those with CAVD was higher than in those without CAVD, which was likely to be responsible for the relatively low serum lipid levels in the patients with CAVD.

\section{Macrophage Infiltration in Noncalcified and Calcific Aortic Valves}

Immunohistochemical staining showed that macrophages infiltration was present in both calcific and noncalcified aortic valves (Figure 1, A). A quantitative analysis of the phenotype of the macrophages showed that the number of infiltrating pan macrophages $\left(\mathrm{CD}^{+} 8^{+}\right)$was significantly larger in calcific aortic valves 

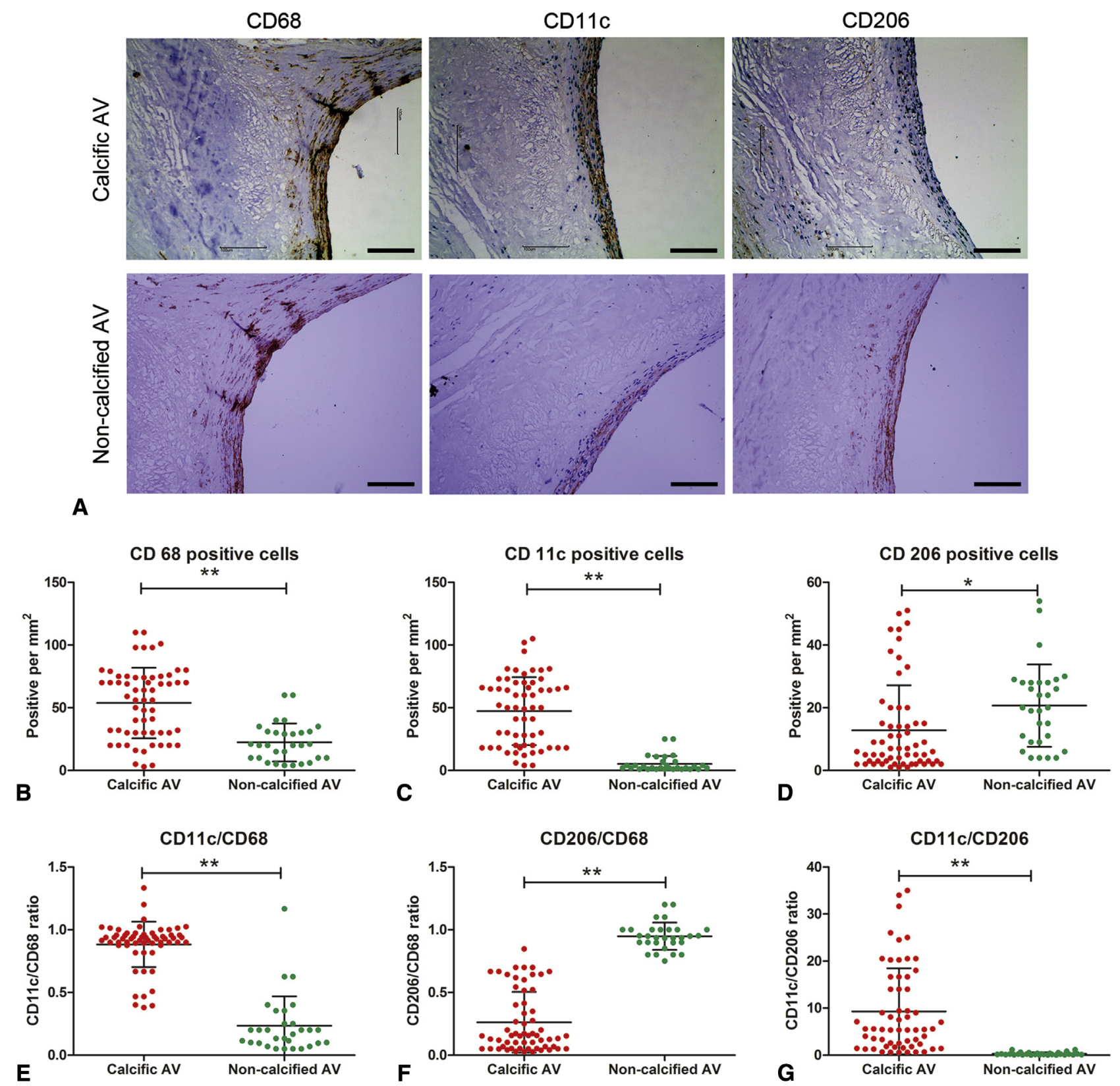

FIGURE 1. Macrophage infiltration in calcific and noncalcified aortic valves. A, Representative images of immunohistochemical staining for CD68, CD11c, and CD206 in the calcific and noncalcified aortic valves. Scale bar $=100 \mu \mathrm{m}$. B-D, Cell counts of infiltrating macrophages. Each point represents the cell count of infiltrating macrophages $\left(/ \mathrm{mm}^{2}\right)$. E-G, M1/M2 macrophage polarization in the calcific and noncalcified aortic valves. Each point represents the ratio of $(\mathrm{E}) \mathrm{CD} 11 \mathrm{c}^{+}$and $(\mathrm{F}) \mathrm{CD} 206^{+}$cells to $\mathrm{CD} 68^{+}$cells and $(\mathrm{G}) \mathrm{CD} 11 \mathrm{c}^{+}$cells to $\mathrm{CD} 206^{+}$cells. Bars represent mean \pm standard deviation. $* P<.05$, $* * P<.01$. Color legend: calcific aortic valves $($ red $)$, noncalcified aortic valves (green). AV, Aortic valve.

than that in noncalcified aortic valves $(P<.01)$ (Figure 1, $B)$. The number of M1 macrophages $\left(\mathrm{CD} 11 \mathrm{c}^{+}\right)$was significantly larger in calcific aortic valves $(P<.01)$ (Figure $1, C$ ). The $\mathrm{CD} 11 \mathrm{c}^{+} / \mathrm{CD} 8^{+}$ratio was significantly higher in calcific aortic valves than in noncalcified aortic valves $(P<.01)$ (Figure $1, E)$. The number of M2 macrophages $\left(\mathrm{CD} 206^{+}\right)$was significantly smaller in calcific aortic valves than in noncalcified aortic valves $(P<.05)$ (Figure $1, D)$. To this effect, the ratio of
CD $206^{+} / \mathrm{CD} 8^{+}$cells was significantly lower in calcific aortic valves than in noncalcified aortic valves $(P<.01)$ (Figure $1, F)$. The ratio of $\mathrm{CD} 11 \mathrm{c}^{+} / \mathrm{CD} 206^{+}$ was significantly increased in calcific aortic valves compared with noncalcified aortic valves $(P<.01)$ (Figure 1, G). These data indicated that the total number of macrophages increased within the human calcific aortic valves and that M1 phenotype was the predominant macrophage population. 
TNF- $\alpha$ mRNA expression
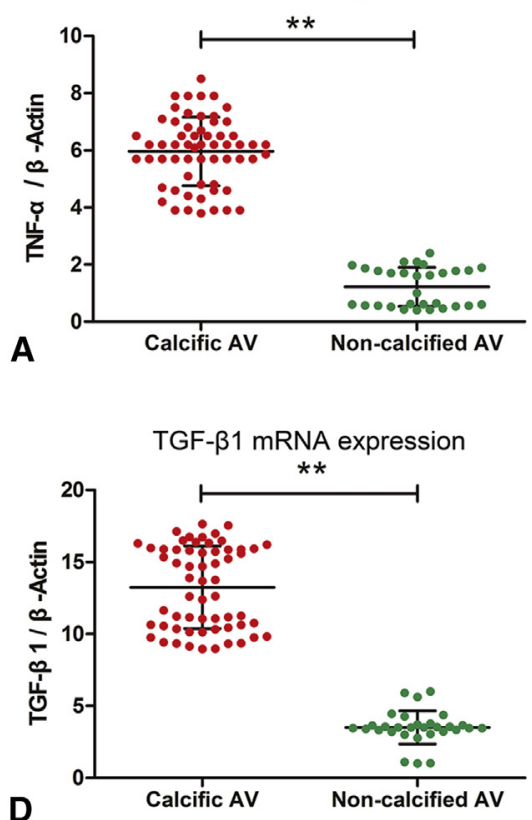

IL-12a mRNA expression

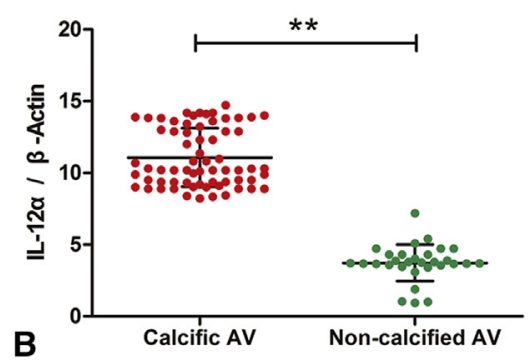

IL-10 mRNA expression

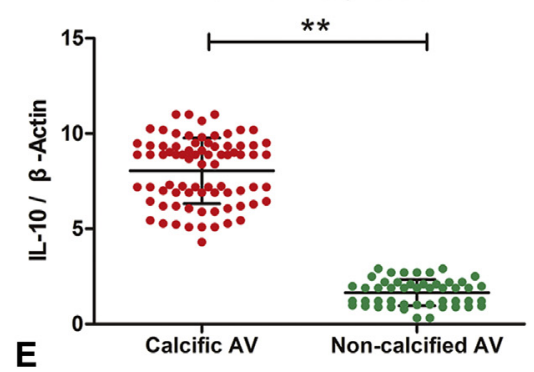

IL-6 mRNA expression

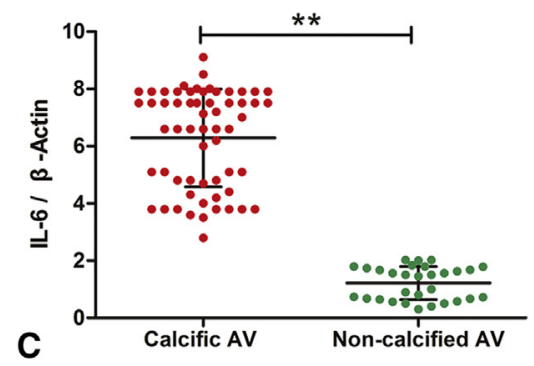

AMAC-1 mRNA expression

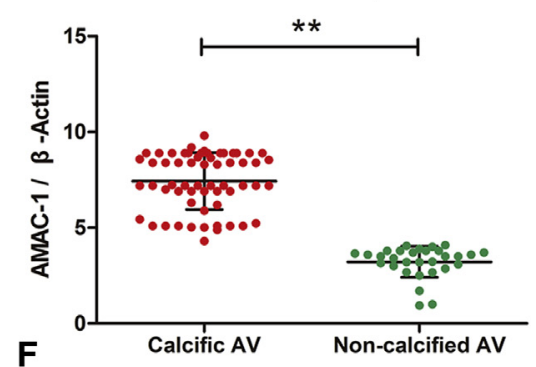

FIGURE 2. Cytokine expression in calcific and noncalcified aortic valves. A-C, mRNA expression of proinflammatory cytokines in calcific and noncalcified aortic valves. Each point represents the expression level assessed by real-time quantitative PCR. Expression level was normalized to each $\beta$-actin level. D-F, mRNA expression of anti-inflammatory cytokines in calcific and noncalcified aortic valves. Bars represent mean \pm standard deviation. ${ }^{* *} P<.01$. Color legend: calcific aortic valves $(r e d)$, noncalcified aortic valves ( green). $T N F$, Tumor necrosis factor; $I L$, interleukin; $A V$, aortic valve; $T G F$, transforming growth factor; $A M A C$, alternative macrophage activation-associated $\mathrm{CC}$ chemokine.

\section{Cytokines Expression in Noncalcified and Calcific Aortic Valves}

To analyze the changes in gene expression levels of M1/M2 cytokines in noncalcified or calcific aortic valves, quantitative real-time PCR reaction was applied. The expression level of proinflammatory cytokines, TNF- $\alpha$, IL-12 $\alpha$, and IL-6, was significantly increased in calcific aortic valves compared with noncalcified aortic valves $(P<.01)$ (Figure $2, A-C)$. The mRNA expression of antiinflammatory cytokines, transforming growth factor- $\beta 1$, IL-10, and alternative macrophage activation-associated CC chemokine-1, were upregulated in calcific aortic valves compared with noncalcified aortic valves $(P<.01)$ (Figure 2, D-F). These results suggested that both proinflammatory and anti-inflammatory cytokines were upregulated in the calcific aortic valves compared with noncalcified aortic valves.

\section{Lipopolysaccharide-induced M1 Polarization of U937 Monocytic Cells}

After phorbol-12-myristate-13-acetate treatment, U937 monocytic cells differentiated into the adherent macrophages. In an effort to better understand the impact of LPS concentration on macrophage differentiation, we used a range of concentrations $(0,100,200$, and $500 \mathrm{ng} / \mathrm{mL}$ ). Quantitative real-time PCR reaction and Western blotting data showed that the mRNA level and protein expression of inducible nitric oxide synthase, the production of M1 macrophage phenotype, was upregulated by LPS treatment in a dose-dependent manner (Figure 3, $A$ and $B$ ). Because no significant difference of inducible nitric oxide synthase expression was found in cells treated with $200 \mathrm{ng} / \mathrm{mL}$ compared with cells treated with $500 \mathrm{ng} / \mathrm{mL}$. The concentration of LPS used in the following experiment was $200 \mathrm{ng} / \mathrm{mL}$. Furthermore, the cell surface expression of the stimulated macrophages was confirmed by flow cytometry. Representative histograms of flow cytometry data in Figure 3, C, showed that LPS-stimulated macrophages presented with high numbers of CD11c-positive macrophages (M1) compared with nonstimulated cells $(96.4 \% \pm 1.2 \%$ to $70.7 \% \pm 3.3 \%, P<.001)$. These findings indicated that the majority of the LPSstimulated macrophages were M1 proinflammatory macrophages.

\section{Highly Expressed Tumor Necrosis Factor- $\alpha$ and Interleukin-6 in Conditioned Medium}

Enzyme-linked immunosorbent assay data demonstrated that the average concentration of TNF- $\alpha$ and IL-6 in conditioned medium from LPS-stimulated macrophages was significantly higher than in conditioned medium from unstimulated macrophages $(P<.001)$ (Figure $3, D$ and $E$ ), which suggested that M1 

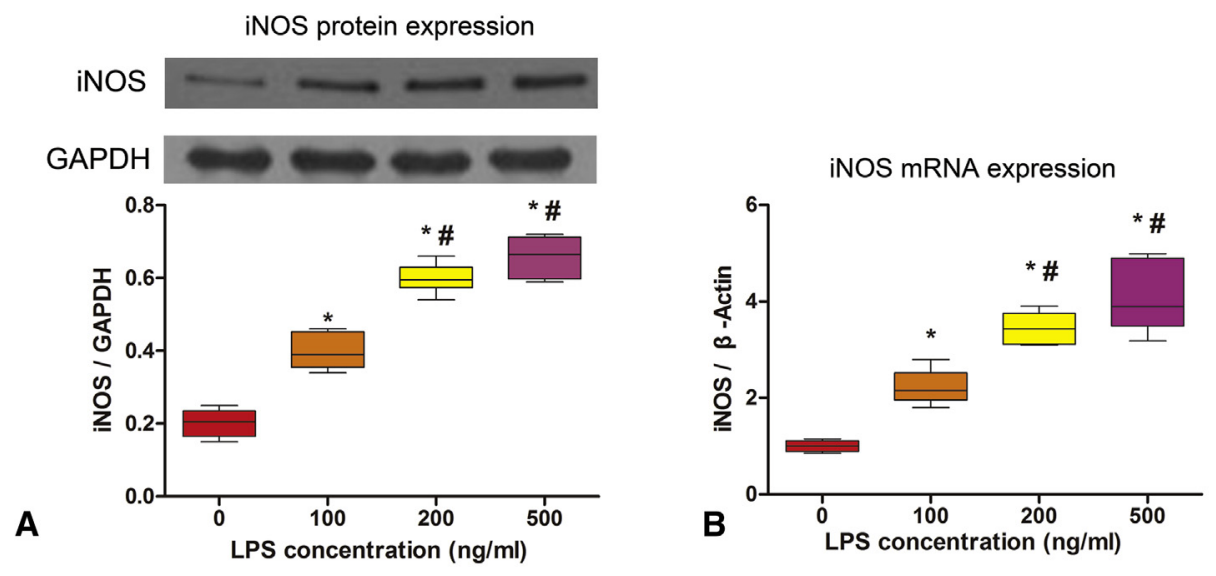

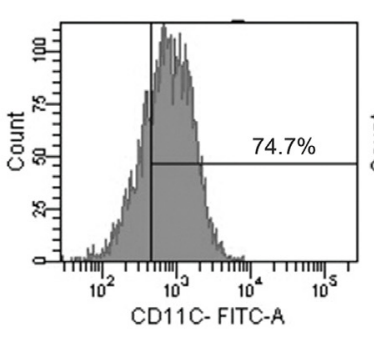

C

LPS $=0$

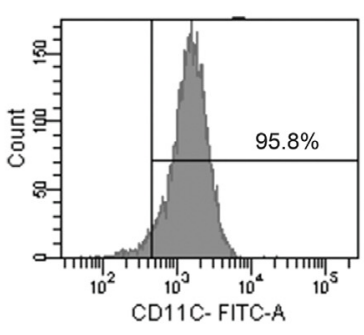

LPS $=\mathbf{2 0 0}$

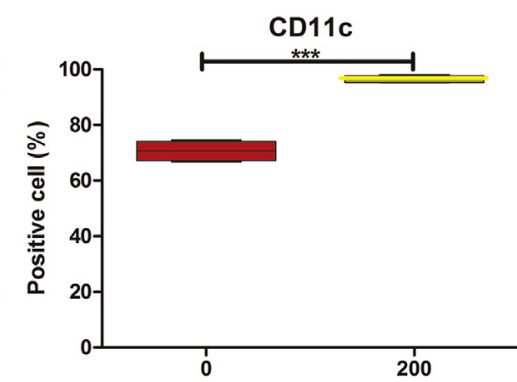

LPS concentration $(\mathrm{ng} / \mathrm{ml})$

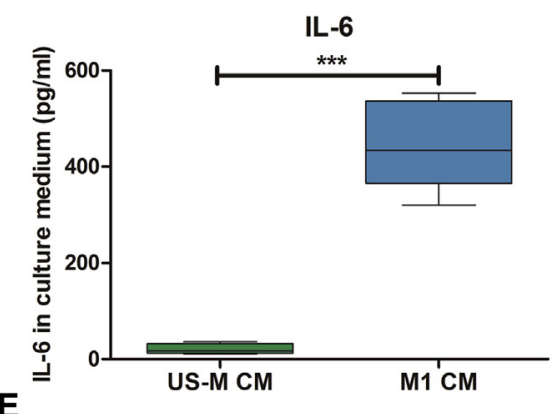

FIGURE 3. A, Representative Western blotting picture and densitometric quantification of inducible nitric oxide synthase expression. B, mRNA expression of inducible nitric oxide synthase assessed by real-time quantitative PCR. The LPS treatment increased the expression of inducible nitric oxide synthase, the production of M1 macrophage phenotype in a dose-dependent manner. $* P<.05$ versus $\mathrm{LPS}=0 \mathrm{ng} / \mathrm{mL}$ group. $\# P<.05$ versus $\mathrm{LPS}=100 \mathrm{ng} / \mathrm{mL}$ group. C, Representative histograms of flow cytometry and positive proportion of CD11 ${ }^{+}$cells in unstimulated or LPS-stimulated macrophages. LPS-stimulated macrophages highly expressed M1 macrophage marker CD11c. $* * * P<.001$. D and E, Enzyme-linked immunosorbent assay of TNF- $\alpha$ and IL-6 in conditioned medium from M1 macrophages or unstimulated macrophages. $* * * P<.001$. Color legend: LPS $=0 \mathrm{ng} / \mathrm{mL}$ (red), LPS $=100 \mathrm{ng} / \mathrm{mL}($ orange), LPS $=200 \mathrm{ng} / \mathrm{mL}$ (yellow), LPS $=500 \mathrm{ng} / \mathrm{mL}$ (purple), conditioned medium from unstimulated macrophage (green), conditioned medium from M1 macrophage (blue). US-M CM, Conditioned medium from unstimulated macrophage; $M 1 C M$, conditioned medium from M1 macrophages; $i N O S$, inducible nitric oxide synthase, GAPDH, glyceraldehyde 3-phosphate dehydrogenase; $L P S$, lipopolysaccharide; $T N F$, tumor necrosis factor.

macrophages highly secreted proinflammatory cytokines, TNF- $\alpha$, and IL- 6 .

\section{Conditioned Medium from M1 Macrophages} Promoted Osteogenic Differentiation of Valvular Interstitial Cells

To investigate the effect of macrophages on the human VICs, VICs from noncalcified aortic valve were incubated with the nonconditioned (fresh) medium or conditioned medium from unstimulated macrophages or M1 macrophages. To prevent residual LPS contamination, conditioned medium was neutralized with polymyxin B before treatment of VICs. Immunofluorescence results showed positive $\alpha$-smooth muscle actin and vimentin expression in VICs cultures, which led us to the conclusion that our isolated VICs were actually true VICs (Figure E1). After 7 days of 
BMP2 mRNA expression

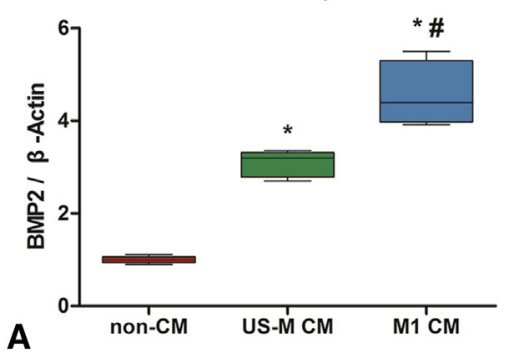

BMP2 protein expression
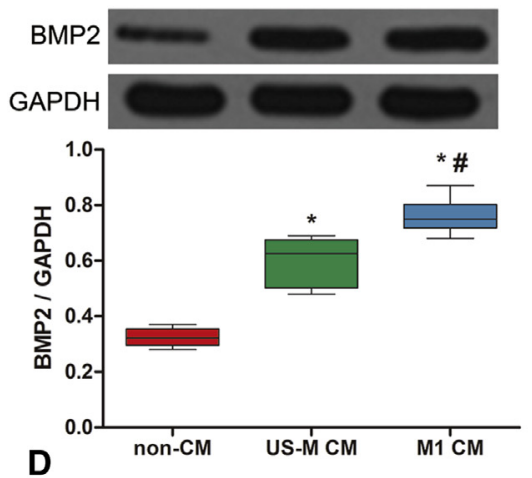

OPN mRNA expression

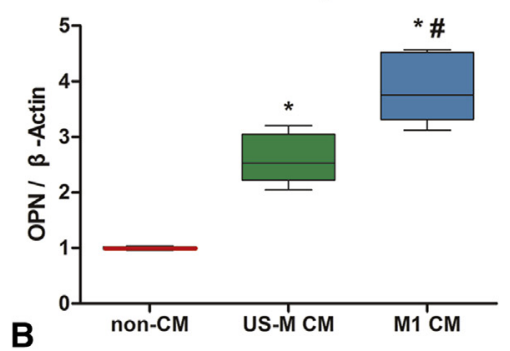

OPN protein expression

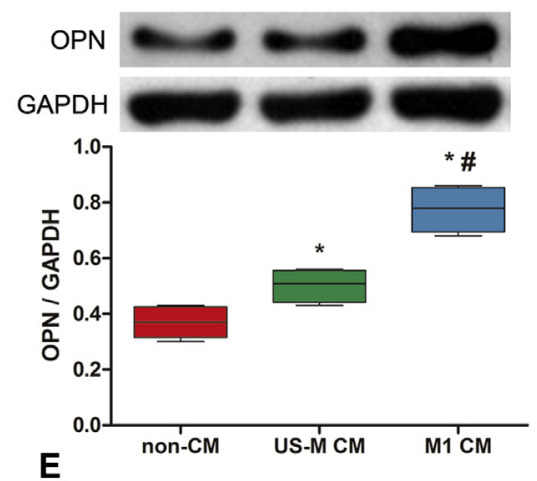

ALP mRNA expression
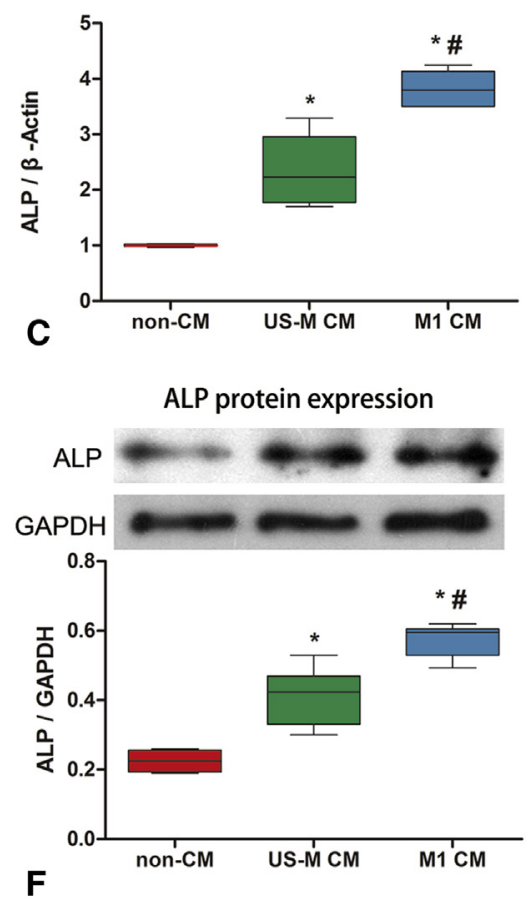

FIGURE 4. Conditioned media from M1 macrophages promoted osteogenic differentiation of VICs. A-C, mRNA expression of osteoblastic markers including BMP2, OPN, and ALP assessed by real-time quantitative PCR. D-F, Representative Western blotting picture and densitometric quantification of osteoblastic markers. $* P<.05$ versus non-CM. $\# P<.05$ versus US-M CM. Color legend: nonconditioned medium (red), conditioned medium from unstimulated macrophage (green), conditioned medium from M1 macrophage (blue). BMP, Bone morphogenetic protein; OPN, osteopontin; ALPALP, alkaline phosphatase; GAPDH, glyceraldehyde 3-phosphate dehydrogenase; US-M CM, conditioned medium from unstimulated macrophage.

incubation, PCR and Western blotting data showed that the mRNA level and protein expression of several osteoblastic markers, including BMP2, ALP, and OPN, significantly increased in VICs with conditioned medium from unstimulated macrophages than in VICs with nonconditioned (fresh) medium (Figure 4, A-F). Conditioned medium from M1 macrophages further enhanced expression of these osteoblastic genes and highly expressed proinflammatory cytokines. When taken together, these results indicated that M1 macrophage might secrete proinflammatory cytokines which promote the calcification in human aortic valves.

\section{Inhibition of Tumor Necrosis Factor- $\alpha$ and Interleukin-6 Attenuated Valvular Interstitial Cell Calcification Induced by Conditioned Medium from M1 Macrophages}

TNF- $\alpha$ and IL-6 are highly increased in conditioned medium from M1 macrophages, and previous studies have shown that TNF- $\alpha$ and IL- 6 might promote the calcification of VICs. Therefore, we examined whether TNF- $\alpha$ and IL-6 are responsible for the osteo-inductive effect of the conditioned medium from M1 macrophages. VICs were incubated with conditioned media from M1 macrophages that had been pretreated for 1 hour with neutralizing antibodies against TNF- $\alpha(1 \mu \mathrm{g} / \mathrm{mL})$ and IL-6 $(1 \mu \mathrm{g} / \mathrm{mL})$, alone or in combination. ${ }^{20}$ Osteoblastic markers were measured after 7 days of culture. As shown in Figure 5, $A-C$, Western blotting data showed that TNF- $\alpha$ or IL-6 neutralizing antibody decreased the induction of osteoblastic markers including BMP2, OPN, and ALP. In addition, inhibiting both TNF- $\alpha$ and IL- 6 appeared to further attenuate osteo-inductive effect of the conditioned medium from M1 macrophages. Moreover, inhibition of TNF- $\alpha$ and IL- 6 attenuated the calcium deposits of VICs (Figure 5,D). These results indicated that the effects of conditioned medium from M1 macrophages on VICs osteogenic differentiation were mediated, at least in part, by TNF- $\alpha$ and IL-6.

\section{DISCUSSION}

In this study, we found that infiltration of macrophages was enhanced in the human calcific aortic valve and M1 phenotype was the predominant macrophage subset according to this Chinese population investigation. In addition, we observed both proinflammatory and antiinflammatory cytokines were significantly upregulated in the calcific aortic valves compared with that in the 

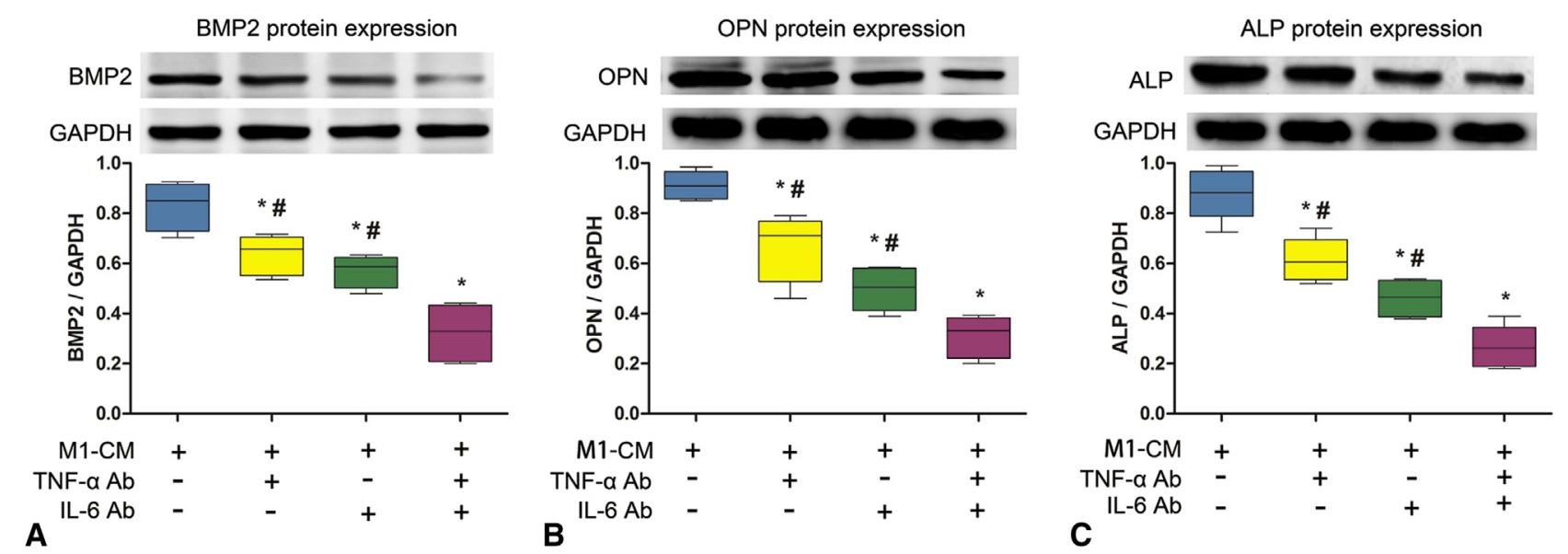

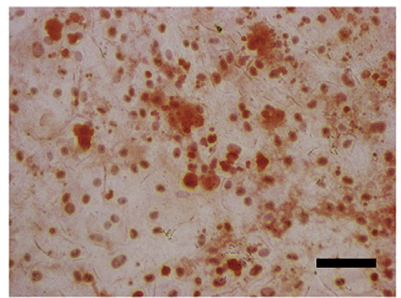

D

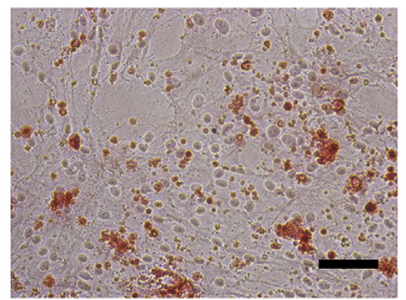

$M 1-C M+T N F-\alpha A b$

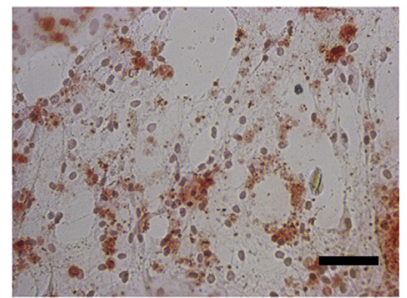

$\mathrm{M} 1-\mathrm{CM}+\mathrm{IL}-6 \mathrm{Ab}$

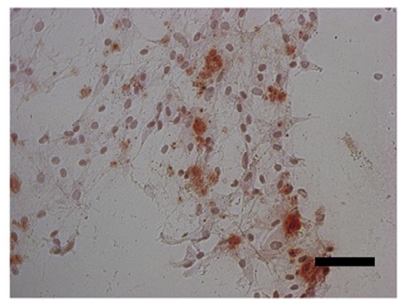

$M 1-C M+T N F-\alpha A b+I L-6 A b$

FIGURE 5. Inhibition of TNF- $\alpha$ and IL-6 attenuated conditioned media from M1 macrophages mediated enhancement of VICs calcification. A-C, Representative Western blotting picture and densitometric quantification of osteoblastic markers including BMP2, ALP, and OPN. $* P<.05$ versus M1-CM. \#P<.05 versus M1-CM + TNF- $\alpha$ antibody + IL-6 antibody. D, Representative alizarin red staining of VICs. Inhibition of TNF- $\alpha$ and IL-6 attenuated the calcium deposits of VICs. Scale bar $=100 \mu \mathrm{m}$. Color legend: conditioned medium from M1 macrophage (blue), conditioned medium from M1 macrophage supplemented with TNF- $\alpha$ antibody (yellow), conditioned medium from M1 macrophage supplemented with IL-6 antibody (green), conditioned medium from M1 macrophage supplemented with TNF- $\alpha$ and IL-6 antibodies (purple). BMP, Bone morphogenetic protein; GAPDH, glyceraldehyde 3-phosphate dehydrogenase; $O P N$, osteopontin; $A L P$, alkaline phosphatase; $M 1$ - $C M$, conditioned medium from M1 macrophage; $T N F$, tumor necrosis factor; $I L$, interleukin.

noncalcified valves. Furthermore, we demonstrated that conditioned medium from unstimulated macrophages promoted the osteogenic differentiation of VICs in vitro, as evidenced by increased osteoblastic markers including BMP2, OPN, and ALP. Conditioned medium from M1 macrophages further enhanced VICs calcification. Neutralizing antibodies to TNF- $\alpha$ and IL-6 attenuated the induction of osteoblastic markers by conditioned medium from M1 macrophage.

CAVD includes a wide range of clinical features from aortic fibrosis, sclerosis to severe stenosis. In the early stages of CAVD, calcification, co-localizing to areas of lipid deposition, consists of nodules containing hydroxyapatite deposited on a bone-like matrix of collagen, OPN, and other bone matrix proteins. ${ }^{21,22}$ Remodeling of this calcification occurs as CAVD progresses until by the end stages of disease, lamellar bone with hematopoietic elements, microfractures, and neoangiogenesis can be observed in the valve. ${ }^{22,23}$ To rule out potentially differences between various stages of CAVD, we classified the calcified aortic valves into 4 grades based on the gross appearance and echocardiography (Table 1). Valves with calcification grade
1 or 2 were used as calcific aortic valves, and valves with calcification grade 0 were considered as noncalcified aortic valves.

In the present study, we showed that the infiltration of macrophages was enhanced in the human calcific aortic valve. A set of proinflammatory and anti-inflammatory macrophages, with extreme polarization of M1 and M2 macrophages, were observed in calcific aortic valves. Of note, M1 macrophages were the predominant phenotype subsets in calcific aortic valves. It suggests that both macrophage numbers and a proinflammatory phenotype influence the calcification of aortic valves. Studies have shown that macrophages possess remarkable functional plasticity and alter functions in response to micro-environmental changes in the pathogenesis of atherosclerosis. ${ }^{14}$ Hirata and colleagues $^{24}$ reported that coronary atherosclerosis was associated with macrophage polarization in epicardial adipose tissue. ${ }^{24}$ CAVD has similar pathological processes and clinical risk factors to atherosclerosis. ${ }^{15}$ Therefore, the results from the present study are consistent with calcific aortic valves also being associated with macrophage polarization. 
In the inflammation-related diseases, macrophages were identified as major contributors to the inflammatory response through their secretion of proinflammatory mediators and anti-inflammatory cytokines. $^{11}$ M1 macrophage expresses numerous proinflammatory mediators including IL-1, IL-6, IL-12, IL-23, TNF- $\alpha$, reactive nitrogen and oxygen intermediates, which help to orchestrate and amplify Th1 immune responses and have a strong microbicidal activity. M2 macrophage phenotype, which is capable of phagocytosis, secretes antiinflammatory cytokines such as IL-1 receptor antagonist, IL-10, transforming growth factor- $\beta 1$, and alternative macrophage activation-associated CC chemokine-1, and induces Th2 immune responses. ${ }^{11-14}$ In this study, we observed that both proinflammatory and antiinflammatory cytokines were significantly upregulated in the calcific aortic valves compared with noncalcified valves, which were in agreement with the increased macrophage number. The increased cytokines, such as TNF- $\alpha$, IL-6, and IL- $1 \beta$, was reported to be associated with increased production of matrix metalloproteinases, which contribute to the extracellular matrix degradation, remodeling, and valvular calcification. ${ }^{25-27}$

During the process of CAVD, VICs in the diseased valves lose their normally quiescent fibroblastic phenotype and undergo myofibroblastic activation. In the calcified lesions, there are mainly osteogenic differentiated VICs expressing a panel of osteoblastic markers. ${ }^{28,29}$ The results from the present study are consistent with the fact that calcific aortic valves are correlated with macrophage polarization and inflammatory response. We used a primary cell co-culture model of human noncalcified aortic VICs with conditioned medium. The results of this in vitro model indicated that unstimulated macrophages promoted osteoblastic differentiation of VICs, as evidenced by increased osteogenic differentiation markers including BMP2, OPN, and ALP. Polarization of M1 macrophages further enhanced VIC calcification. This is in accordance with the report of Tintut and colleagues ${ }^{17}$ that macrophages enhanced vascular calcification in vitro, and LPS or oxidized LDL may further promote vascular cell calcification by activating macrophages to induce osteoblastic differentiation.

The mechanisms by which M1 macrophage promote the calcification of the aortic valve are complex, but the effect of LPS activation was mediated at least in part through secretion of TNF- $\alpha$ and IL- 6 . An increased expression of the TNF- $\alpha$ and IL- 6 has been shown in calcific aortic valves and in vitro monocyte-related media incubated with LPS. Our results indicated that conditioned medium from M1 macrophages enhanced osteogenic differentiation of VICs. Thus, M1 macrophage phenotype may, by secretion of TNF- $\alpha$ and IL-6, promote an inflammatory microenvironment in calcific aortic valves that in turn leads to osteogenic differentiation of VICs. Previous studies have shown that TNF- $\alpha$ might be one important mediator that upregulate the expression of BMP2 and promote the calcification of VICs with activation of nuclear factor$\kappa \mathrm{B} \cdot{ }^{25,30}$ IL-6 also was shown to promote the expression of BMP2 that increased mineralization of VICs. ${ }^{31}$ In our co-culture model, the functions of TNF- $\alpha$ and IL- 6 had been confirmed by blocking with neutralizing antibody.

\section{Study Limitations}

First, a limited number of surface markers were used for determination of the M1 or M2 phenotype of the macrophages participating in the process of valve calcification. Second, LPS induced macrophage polarization in vitro could not completely imitate the macrophage polarization process of CAVD in vivo. Third, the interaction between M1 and VICs remains unknown, and further work is needed to better understand the function of macrophage polarization on VICs calcification. Finally, sample numbers in this clinic study were not sufficient to investigate the cause in more detail.

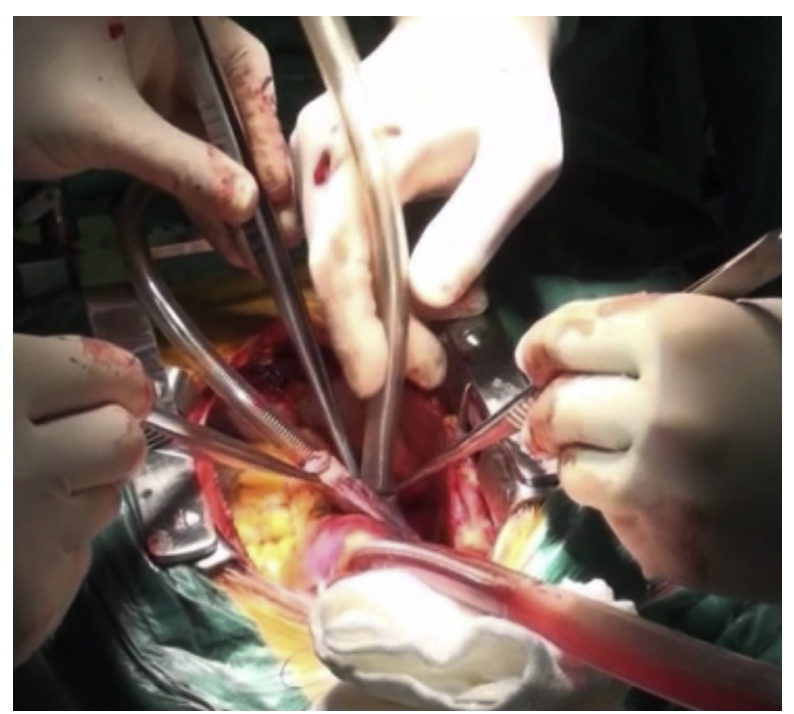

VIDEO 1. A surgery procedure is shown. The patient is a 68 -year-old man with aortic valve stenosis and coronary artery disease for years. He is receiving aortic valve replacement and CABG together. As we noted in the article, approximately $38.33 \%$ of the patients with CAVD in our study and a coronary heart disease have major morbidity, mortality, and health economic costs. CAVD is the most common form of heart disease and a major healthcare burden. Our present study may give us more information about the macrophage subsets and their functions in CAVD. Despite the high prevalence of CAVD, there is still no highly effective nonsurgical therapeutic strategy or prevention method at present. Targeting macrophage polarization and altering their phenotype to adapt to the microenvironment might be promising for these patients. Video available at: http://www.jtcvsonline.org/article/S0022-5223(17)30202-7/addons. 


\section{CONCLUSIONS}

The results of the present study demonstrate that both macrophage numbers and polarization influence the process of human aortic valve calcification. Macrophage polarization may interplay with VICs through secretion of proinflammatory mediators to promote osteogenic differentiation of VICs. Additional in vivo and human studies are required to extend the relevance of these data to the human condition. Despite the high prevalence of CAVD, there is still no highly effective nonsurgical therapeutic strategy or prevention method at present (Video 1). Targeting macrophage polarization and altering their phenotype to adapt to the microenvironment might be promising for them.

\section{Conflict of Interest Statement}

Authors have nothing to disclose with regard to commercial support.

\section{References}

1. Rajamannan NM, Evans FJ, Aikawa E, Grande-Allen KJ, Demer LL, Heistad DD, et al. Calcific aortic valve disease: not simply a degenerative process a review and agenda for research from the National Heart and Lung and Blood Institute Aortic Stenosis Working Group Executive Summary: Calcific Aortic Valve Disease-2011 Update. Circulation. 2011;124:1783-91.

2. Pan W, Zhou D, Cheng L, Shu X, Ge J. Candidates for transcatheter aortic valve implantation may be fewer in China. Int J Cardiol. 2013;168:e133-4.

3. LeMaire SA. Individualized treatment strategies for patients with aortic valve disease and porcelain aorta. J Thorac Cardiovasc Surg. 2015;149:134-6.

4. Mathieu P, Boulanger MC. Basic mechanisms of calcific aortic valve disease. Can J Cardiol. 2014;30:982-93.

5. Bosse Y, Miqdad A, Fournier D, Pépin A, Pibarot P, Mathieu P. Refining molecular pathways leading to calcific aortic valve stenosis by studying gene expression profile of normal and calcified stenotic human aortic valves. Circ Cardiovasc Genet. 2009;2:489-98.

6. Steiner I, Krbal L, Rozkoš T, Harrer J, Laco J. Calcific aortic valve stenosis: immunohistochemical analysis of inflammatory infiltrate. Pathol Res Pract. 2012;208:231-4.

7. Coté N, Mahmut A, Bosse Y, Couture C, Pagé S, Trahan S, et al. Inflammation is associated with the remodeling of calcific aortic valve disease. Inflammation. 2013;36:573-81.

8. Moreno PR, Astudillo L, Elmariah S, Purushothaman KR, Purushothaman M, Lento PA, et al. Increased macrophage infiltration and neovascularization in congenital bicuspid aortic valve stenosis. J Thorac Cardiovasc Surg. 2011;142:895-901.

9. Wada S, Sugioka K, Naruko T, Kato Y, Shibata T, Inoue T, et al. Relationship between oxidative stress and aortic valve stenosis in humans: an immunohistochemical study. Osaka City Med J. 2013;59:61-7.

10. Classen A, Lloberas J, Celada A. Macrophage activation: classical versus alternative. Methods Mol Biol. 2009;531:29-43.

11. Liu YC, Zou XB, Chai YF, Yao YM. Macrophage polarization in inflammatory diseases. Int J Biol Sci. 2014;10:520-9.

12. Mantovani A, Biswas SK, Galdiero MR, Sica A, Locati M. Macrophage plasticity and polarization in tissue repair and remodelling. J Pathol. 2013;229: 176-85.
13. Moore KJ, Sheedy FJ, Fisher EA. Macrophages in atherosclerosis: a dynamic balance. Nat Rev Immunol. 2013;13:709-21.

14. Leitinger N, Schulman IG. Phenotypic polarization of macrophages in atherosclerosis. Arterioscler Thromb Vasc Biol. 2013;33:1120-6.

15. Freeman RV, Otto CM. Spectrum of calcific aortic valve disease pathogenesis, disease progression, and treatment strategies. Circulation. 2005;111:3316-26.

16. Wang B, Zhang C, Wei G, Liao P, Li F, Dong N. High mobility group box-1 protein induces the osteogenic phenotype changes in aortic valve interstitial cells. J Thorac Cardiovasc Surg. 2016;151:255-62.

17. Tintut Y, Patel J, Territo M, Saini T, Parhami F, Demer LL. Monocyte/ macrophage regulation of vascular calcification in vitro. Circulation. 2002; 105:650-5.

18. Chen JH, Yip CY, Sone ED, Simmons CA. Identification and characterization of aortic valve mesenchymal progenitor cells with robust osteogenic calcification potential. Am J Pathol. 2009;174:1109-19.

19. Nadlonek NA, Weyant MJ, Jessica AY, Cleveland JC, Reece TB, Meng X, et al Radiation induces osteogenesis in human aortic valve interstitial cells. J Thorac Cardiovasc Surg. 2012;144:1466-70.

20. Deuell KA, Callegari A, Giachelli CM, Rosenfeld ME, Scatena M. RANKL enhances macrophage paracrine pro-calcific activity in high phosphate-treated smooth muscle cells: dependence on IL-6 and TNF- $\alpha$. J Vasc Res. 2012;49: 510-21.

21. Yu PJ, Skolnick A, Ferrari G, Heretis K, Mignatti P, Pintucci G, et al. Correlation between plasma osteopontin levels and aortic valve calcification: potential insights into the pathogenesis of aortic valve calcification and stenosis. $J$ Thorac Cardiovasc Surg. 2009;138:196-9.

22. Dweck MR, Boon NA, Newby DE. Calcific aortic stenosis: a disease of the valve and the myocardium. J Am Coll Cardiol. 2012;60:1854-63.

23. Mohler ER III, Gannon F, Reynolds C, Zimmerman R, Keane MG, Kaplan FS Bone formation and inflammation in cardiac valves. Circulation. 2001;103: 1522-8.

24. Hirata Y, Tabata M, Kurobe H, Motoki T, Akaike M, Nishio C, et al. Coronary atherosclerosis is associated with macrophage polarization in epicardial adipose tissue. J Am Coll Cardiol. 2011;58:248-55.

25. Kaden JJ, Kiliç R, Sarikoç A, Hagl S, Lang S, Hoffmann U, et al. Tumor necrosis factor alpha promotes an osteoblast-like phenotype in human aortic valve myofibroblasts: a potential regulatory mechanism of valvular calcification. Int J Mol Med. 2005;16:869-72.

26. Yao Y, Watson AD, Ji S, Boström KI. Heat shock protein 70 enhances vascular bone morphogenetic protein-4 signaling by binding matrix Gla protein. Circ Res. 2009;105:575-84

27. Isoda K, Matsuki T, Kondo H, Iwakura Y, Ohsuzu F. Deficiency of interleukin-1 receptor antagonist induces aortic valve disease in BALB/c mice. Arterioscler Thromb Vasc Biol. 2010;30:708-15.

28. Rajamannan NM, Subramaniam M, Rickard D, Stock SR, Donovan J, Springett M, et al. Human aortic valve calcification is associated with an osteoblast phenotype. Circulation. 2003;107:2181-4.

29. Rajamannan NM. Oxidative-mechanical stress signals stem cell niche mediated Lrp5 osteogenesis in eNOS-/- null mice. J Cell Biochem. 2012;113:1623-34.

30. Yu Z, Seya K, Daitoku K, Motomura S, Fukuda I, Furukawa K. TNF- $\alpha$ accelerates the calcification of human aortic valve interstitial cells obtained from patients with calcific aortic valve stenosis via the BMP2-Dlx5 pathway. $J$ Pharmacol Exp Ther. 2011:337:16-23.

31. El Husseini D, Boulanger MC, Mahmut A, Bouchareb R, Laflamme MH Fournier D, et al. P2Y2 receptor represses IL-6 expression by valve interstitial cells through Akt: implication for calcific aortic valve disease. J Mol Cell Cardiol. 2014;72:146-56.

Key Words: calcific aortic valve disease, valve interstitial cell, calcification, macrophage polarization 

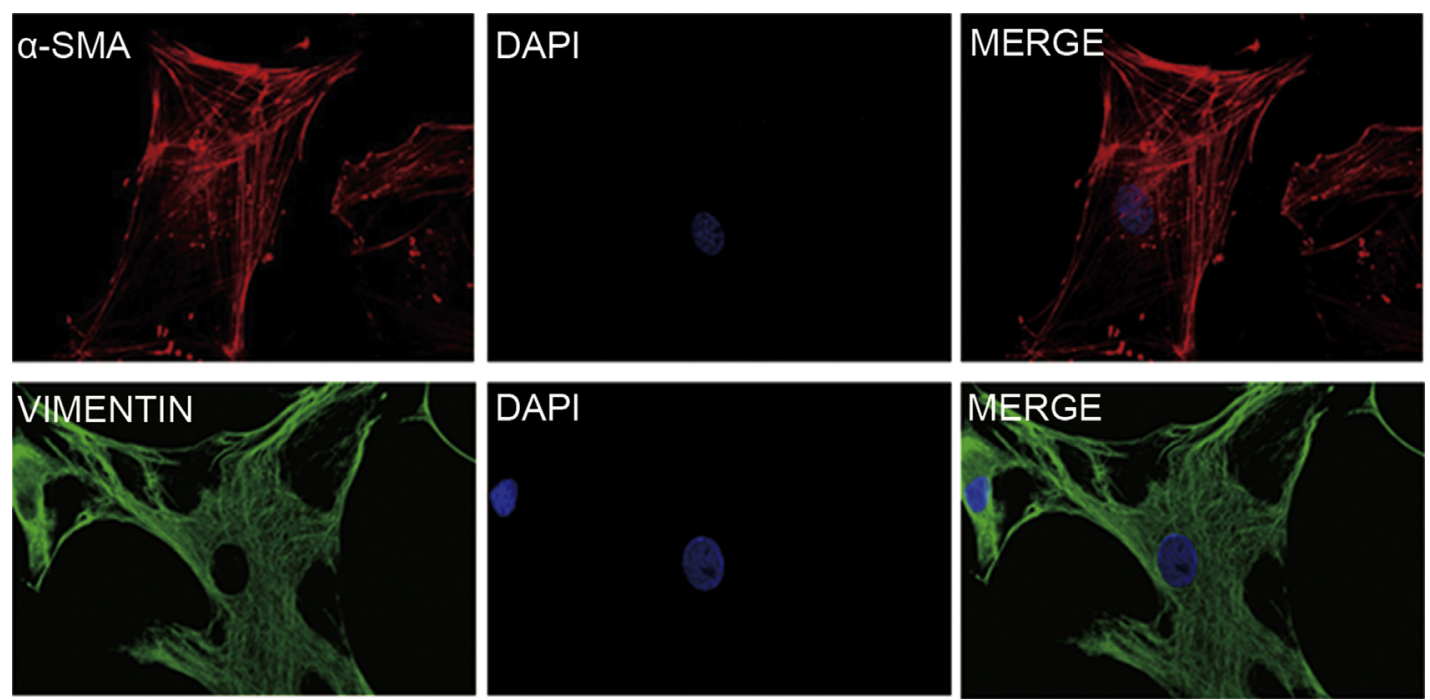

FIGURE E1. Identification of VICs cultured in vitro. Immunofluorescence stainings showed positive $\alpha$-smooth muscle actin and vimentin expression in VIC cultures.

TABLE E1. Primers used for real-time polymerase chain reaction analysis

\begin{tabular}{|c|c|}
\hline Gene & PCR primer sequences $\left(5^{\prime}\right.$ to $\left.3^{\prime}\right)$ \\
\hline \multirow[t]{2}{*}{ IL- $12 \alpha$} & Fw: 5'-GATGGCCCTGTGCCTTAGTA-3' \\
\hline & Rv: 5'-TCAAGGGAGGATTTTTGTGG-3' \\
\hline \multirow[t]{2}{*}{ IL-6 } & Fw: $5^{\prime}$-ATGAGGAGACTTGCCTGGTG-3' \\
\hline & Rv: 5'-CAGGGGTGGTTATTGCATCT-3' \\
\hline \multirow[t]{2}{*}{ IL-10 } & Fw: 5'-GACTTTAAGGGTTACCTGGGTTG-3' \\
\hline & Rv: 5'-TCACATGCGCCTTGATGTCTG-3' \\
\hline \multirow[t]{2}{*}{ AMAC-1 } & Fw: 5'-AGCTCTGCTGCCTCGTCTAT-3' \\
\hline & Rv: 5'-CCCACTTCTTATTGGGGTCA-3' \\
\hline \multirow[t]{2}{*}{ TGF- $\beta 1$} & Fw: $5^{\prime}$-TGCTTCAGCTCCACAGAGAA-3' \\
\hline & Rv: $5^{\prime}$-TGGTTGTAGAGGGCAAGGAC-3' \\
\hline \multirow[t]{2}{*}{ TNF- $\alpha$} & Fw: 5'-TCAGAGGGCCTGTACCTCAT-3' \\
\hline & Rv: 5'-GGAAGACCCCTCCCAGATAG-3' \\
\hline \multirow[t]{2}{*}{$\beta$-actin } & Fw: 5'-CACGATGGAGGGGCCGGACTCATC-3' \\
\hline & Rv: 5'-TAAAGACCTCTATGCCAACACAGT - $3^{\prime}$ \\
\hline \multirow[t]{2}{*}{ iNOS } & Fw: 5'-ACAAGCCTACCCCTCCAGAT- $3^{\prime}$ \\
\hline & Rv: 5'-TCCCGTCAGTTGGTAGGTTC-3' \\
\hline \multirow[t]{2}{*}{ BMP2 } & Fw: $5^{\prime}$-TCAAGCCAAACACAAACAGC $-3^{\prime}$ \\
\hline & Rv: 5'-GGGAGCCACAATCCAGTCAT - -3' \\
\hline \multirow[t]{2}{*}{ ALP } & Fw: $5^{\prime}$-ACATCGCTACGCAGCTCATC $-3^{\prime}$ \\
\hline & Rv: 5'-CCCACCTTGGCTGTAGTCAT - $3^{\prime}$ \\
\hline \multirow[t]{2}{*}{ OPN } & Fw: 5'-CTG AGG AAA AGC AGA ATG-3' \\
\hline & Rv: 5'-AAT GGA GTC CTG GCT GT-3' \\
\hline
\end{tabular}

TABLE E2. Clinical characteristics of the selected patients

\begin{tabular}{|c|c|c|c|}
\hline & $\begin{array}{c}\text { CAVD } \\
(\mathbf{n}=60)\end{array}$ & $\begin{array}{l}\text { Control } \\
(\mathbf{n}=\mathbf{3 0})\end{array}$ & $\begin{array}{c}P \\
\text { value }\end{array}$ \\
\hline Age (y) & $65.95 \pm 8.54$ & $44.17 \pm 12.92$ & $<.001$ \\
\hline Male $(\%)$ & 55 & 76.67 & NS \\
\hline BMI $\left(\mathrm{kg} / \mathrm{m}^{2}\right)$ & $25.58 \pm 2.43$ & $24.07 \pm 2.58$ & $<.01$ \\
\hline Hypertension (\%) & 48.33 & 16.67 & $<.01$ \\
\hline Diabetes mellitus (\%) & 21.67 & 6.67 & NS \\
\hline Coronary artery disease $(\%)$ & 38.33 & 6.67 & $<.01$ \\
\hline Triglycerides (mmol/L) & $1.66 \pm 1.22$ & $1.10 \pm 0.72$ & NS \\
\hline $\mathrm{LDL}(\mathrm{mmol} / \mathrm{L})$ & $2.09 \pm 0.66$ & $2.05 \pm 0.69$ & NS \\
\hline $\mathrm{HDL}(\mathrm{mmol} / \mathrm{L})$ & $1.20 \pm 0.33$ & $1.29 \pm 0.31$ & NS \\
\hline Creatinine $(\mathrm{mmol} / \mathrm{L})$ & $94.87 \pm 25.40$ & $104.8 \pm 31.05$ & NS \\
\hline ACEI $(\%)$ & 38.33 & 40 & NS \\
\hline ARB $(\%)$ & 15 & 16.67 & NS \\
\hline Statin $(\%)$ & 40 & 10 & $<.01$ \\
\hline$\beta$-blocker (\%) & 53.33 & 60 & NS \\
\hline
\end{tabular}

